\title{
Quantitative Thermography in Breast Cancer Detection
}

\author{
by M. Zavisek*, A. Drastich* and J. Sandera* \\ *Brno University of Technology, Brno, Czech Republic
}

\begin{abstract}
This is a research effort that helps to define thermal infrared imaging as a diagnostic tool in breast cancer detection, which can be used as a complementary modality to traditional mammography. Our approach consists in the non-topological and the hybrid methods of computerized thermogram classification. The principle task is to find the most significant thermopathological features.

\section{Introduction}

The breast cancer is the second most often cancerous cause of death among women and the second most frequent type of cancer at all. Nevertheless it is proved that early stages of breast cancer are well treatable. That is why the early detection is so important. Thermography is non-invasive and has proved ability to detect physiological changes caused by early cancer growth. Our approach to thermography application consists in quantitative computer performed analysis and classification of digital thermograms of breasts. This paper summarizes our existing research results.
\end{abstract}

\section{Methods}

Each examination consists of three images. Patient sits on a chair and after twenty minutes long equilibration within air-conditioned room one frontal and two slightly lateral pictures are taken. The analysis is performed in the frontal picture. Each thermogram is attended with finding from X-ray mammography or ultrasound. Digital infrared camera FLIR PM575 is used for thermogram acquisition.

The principal task of our research is to define thermo-pathological features and find the feasible image analysing methods to extract them. As was presented in [1] and many other publications, the differences in vascular pattern and in hot (cold) spots appearance between left and right breast are the most significant thermopathological features. It represents symmetry evaluation of pairs of pictures from our point of view.

\subsection{Image pre-processing}

There are several image pre-processing methods we use. A hot spots and veins should be detected in the image, so it seems to be a good idea to enhance significant thermal gradients (edges) in the image. This operation may be practically realized by derivation. We are not interested in the direction of the edge, so an isotropic version of derivation was devised. We have tested set of directional convolution masks, Laplace operator and sharpening mask performing addition of Laplace filter and original image. The other methods such as averaging (to suppress details and noise) or edge extraction didn't prove to be valuable.

Second pre-analysing adjustment consists in re-sampling of region of interest (ROI - covering the left and right breast) to polar coordinates. The reason is that most of used analysis methods evaluate symmetry of ROI and some of them need them to be registered and with the same number pixels in compared areas. Re-sampling converts symmetry evaluation problem to similarity evaluation. 


\subsection{Feature extraction}

There are two possible techniques of feature extraction - non-topological (statistical) and topology-based methods. Commonly used subjective analysis performed by physician is topology-based [1], but it is not easy to be automated. We do not use any pure topological method, but most of used methods combine both approaches.

\subsubsection{Statistical methods}

Since the cancer creates a new vascular pattern, the overall skin temperature of the affected breast may be higher. The metabolic activity of the cancer tissue is higher, what also increases the overall temperature. The simplest criterion is therefore to compute average temperatures of ROIs and use the absolute value of their difference as a feature. We can get similar results by using median instead of average. The maximum was tested, too. We also compute standard deviation (expected to be higher for breast with vascular pattern) and so called Index 90, which is the " $90 \%{ }^{\text {th" }}$ member of ordered set (similarly the median may be called Index 50 ).

\subsubsection{Histogram-based methods}

Histogram is commonly used technique in image analysis so we just briefly describe the concept and the extracted features, without the mathematical background, which would be redundant in this case.

Two normalized histograms are constructed, one for each breast's ROI. Such histograms should normally be the same for both sides and their difference histogram should have zero values. We extract four features from difference histogram absolute value of its maximum, number of columns exceeding threshold $t$ ( $t=0.01$ in our case), energy and number of zero crosses. We also compute cross correlation function of the both histograms and we use difference of areas under its positive and negative part as a feature.

\subsubsection{Cross Co-Occurrence Matrix}

To understand the principle of this method we need to explain the concept of co-occurrence matrix. Let's have a real matrix (or any two-dimensional shape) $M$. The co-occurrence matrix is matrix $X$ (with elements $x_{k, l}$ ), where each row and each column represent a bin number. Let's assume the bins on both axis are the same $B_{i}$, $\forall i \in \overline{1, M}$. Let's take a pair of pixels (let's name the pixels $a$ and $b$ and their values $m_{a}$ and $m_{b}$ ) from the $M$ with some (usually spatial) relationship. The co-occurrence matrix $X$ includes in individual elements the counts of such pairs, where a belongs to the corresponding "row" bin and $b$ to the "column" one. Expressed mathematically, we may write

$$
x_{k, l}=\operatorname{count}\left\{(a, b) \mid m_{a} \in B_{k} \cap m_{b} \in B_{l}\right\} .
$$

In deed, we can calculate any number of these matrixes - there are many different relationships between the pair of pixels $a$ and $b$ possible. In the special case where $a$ and $b$ are identical, we obtain a diagonal matrix and the elements on main diagonal form the histogram of the $M$.

The cross co-occurrence matrix is defined the same way, but we have two overlapping matrixes (or shapes) $\mathrm{M}$ and $\mathrm{N}$ and pixel $\mathrm{b}$ is taken from the second matrix $\mathrm{N}$ instead from the same matrix (figure 1). Only the special case with zero offset was used, so we may say pixels $a$ and $b$ are on the corresponding place in both shapes. If the shapes of $\mathrm{M}$ and $\mathrm{N}$ are not the same, only overlapping region must be used, or they must be registered (we solved this by re-sampling to polar coordinates). The matrix is normalized by the number of pixels of overlapping region before feature extraction, so the sum of whole matrix is equal to one. 


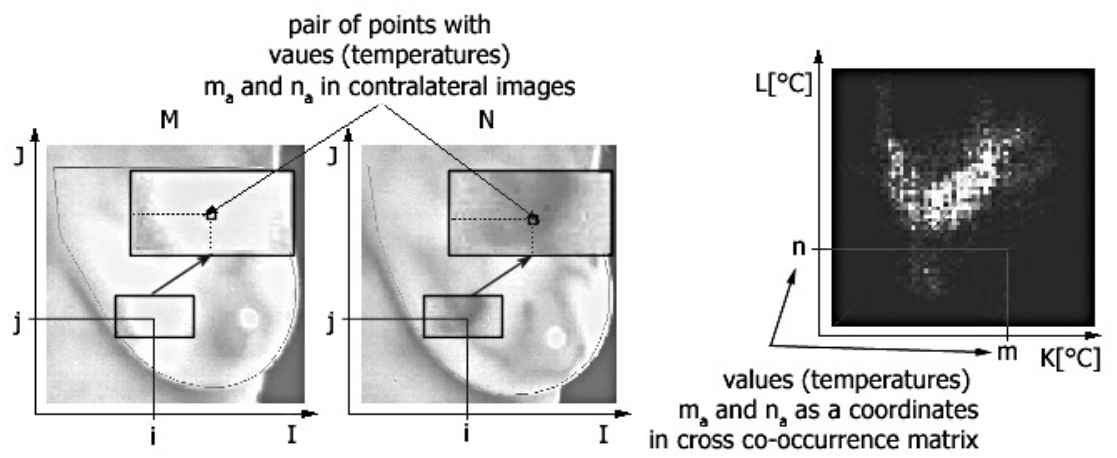

Fig. 1. Concept of the cross co-occurrence matrix

There are many possibilities how to extract features from cross co-occurrence matrix. Let's briefly resume the equations of used features.

Homogeneity of co-occurrence matrix is defined by equation:

$$
G=\sum_{k} \sum_{l} \frac{x_{k, l}}{1+|k-l|}
$$

The interpretation of this feature is that it is the sum of co-occurrence matrix values weighted by the inverse of the distance from the axis - point more distant from the axis has less weight. Since negative thermograms have non-zero elements closer to diagonal, they would have higher homogeneity.

The co-occurrence matrix energy is defined similarly to histogram energy as the sum of squares of normalized co-occurrence matrix values

$$
E=\sum_{k} \sum_{n} x_{k, l}{ }^{2}
$$

The co-occurrence matrix values should be for negative patients around the diagonal axis, so axis moments of different orders should be good features. The axis moment of the $\mathrm{p}^{\text {th }}$ order is defined as Axis moments:

$$
m_{p}=\sum_{k} \sum_{l}(k-l)^{p} x_{k, l}
$$

The $m_{0}$ is always one; the absolute values of moments from $m_{1}$ to $m_{4}$ are used as features.

Other useful features are contrasts (there are two possible definitions)

$$
\begin{aligned}
& C=\sum_{k} \sum_{l}|k-I| x_{k, I}, \\
& C^{\prime}=\sum_{l} \sum_{k>l}|k-I|\left|x_{k, l}-x_{l, k}\right|,
\end{aligned}
$$

and symmetry:

$$
S=1-\sum_{l} \sum_{k>l}\left|x_{k, l}-x_{l, k}\right| .
$$

It's clear that that perfectly symmetrical matrix has the symmetry of one, all non-symmetric matrixes smaller. Negative patients would have, therefore, higher symmetry then positive ones. 


\subsubsection{Mutual information and Joint entropy}

There is a statistical parameter, which describes statistical (in)dependence of two (or more) sets of data, it is called mutual Information (MI). It can be used for evaluation of the similarity of the left and the right breast pattern. It is small for completely independent (or non-similar) sets of data - positive patients would have small values of MI. Similarly the joint entropy $H$ represents the relationship between two sets of data - the negative value of joint entropy is taken as feature, since entropy is always negative. The mutual information can be calculated by the equation

$$
M I=H_{L}+H_{R}-H
$$

where $H_{L}, H_{R}$ and $H$ are entropies of left and right side and the joint entropy defined

$$
\begin{aligned}
& H_{L}=-\sum_{\forall k} p_{L}(k) \log _{2} p_{L}(k) \\
& H_{R}=-\sum_{\forall I} p_{R}(I) \log _{2} p_{R}(I) \\
& H=-\sum_{\forall k} \sum_{\forall I} p_{L R}(k, I) \log _{2} p_{L R}(k, I)
\end{aligned}
$$

where: $p_{R}(l) \ldots$ is the probability density that the random value $R$ equals $I$,

$p_{L}(k) \ldots$ is the probability density that the random value $L$ equals $k$, and

$p_{L R}(k, l)$. is the probability density that the random value $R$ equals $k$ and value $R$ equals $l$.

These probabilities may be estimated from cross co-occurrence matrix according following equations

$$
p_{L R}(k, I)=\frac{x_{k, I}}{\sum_{\forall k, I} x(k, I)}, p_{L}(k)=\sum_{\forall I} p_{L R}(k, I), p_{R}(I)=\sum_{\forall k} p_{L R}(k, I) \quad(12)(13)(14)
$$

\subsubsection{Moments Analysis}

Set of raw moments of ROIs transformed to polar coordinates is computed. The raw moments are defined by equation:

$$
m_{p q}=\int_{-\infty}^{\infty} \int_{-\infty}^{\infty} x^{p} y^{q} f(x, y) d x d y \quad p, q=1,2,3 \ldots
$$

Moment $m_{00}$ is basically only an integral over the whole area. Negative patients should have similar left and right breast and their difference should be small and with varying sign. The moments $m_{01}$ and $m_{10}$ are proportional to coordinates of centre of gravity (COG). The distance of COG from the geometrical centre of breast is another feature, which seems to be very promising for classification. Patients with breast cancer have small distance of COG from the centre. This is probably caused by the fact that negative patients have usually some part of breast a little bit warmer or colder and this causes the move of COG, while the positive patients with strong vascular pattern have often the difference of temperature oscillating around zero - the COG remains approximately at the centre. 


\subsubsection{Fourier analysis}

Fourier spectrum analysis also seems to be good tool for feature extraction, because the warm lines and spots should be visible as higher spatial frequencies in the spectrum. It was computed in polar coordinates too. The mathematical background is commonly known so we can skip it. For extraction of two features we use the difference of absolute values (modulus) of the ROIs spectrums. The features are a value of the difference maximum and distance of this maximum from the centre.

\subsection{Classification}

The next step is to classify images into positive, negative and (possibly) suspicious classes according to selected features. To successfulness evaluation we use three parameters.

Sensitivity is the ratio of positively detected patients and all positive patients

$$
\text { Sensitivity }=\frac{\text { Number of true positive patiens }}{\text { Number of true positive patiens }+ \text { Number of false negative patiens }}
$$

Specificity is similarly

$$
\text { Specificity }=\frac{\text { Number of true negative patiens }}{\text { Number of true negative patiens }+ \text { Number of false positive patiens }}
$$

These parameters are dependent on the value of threshold we adjust to separate the positive and negative patients. We have extracted more than 30 features, including the features derived using preprocessing methods [2]. We selected those 12 features from them, which have intersection of sensitivity and specificity higher then $60 \%$ (figure 2 ).

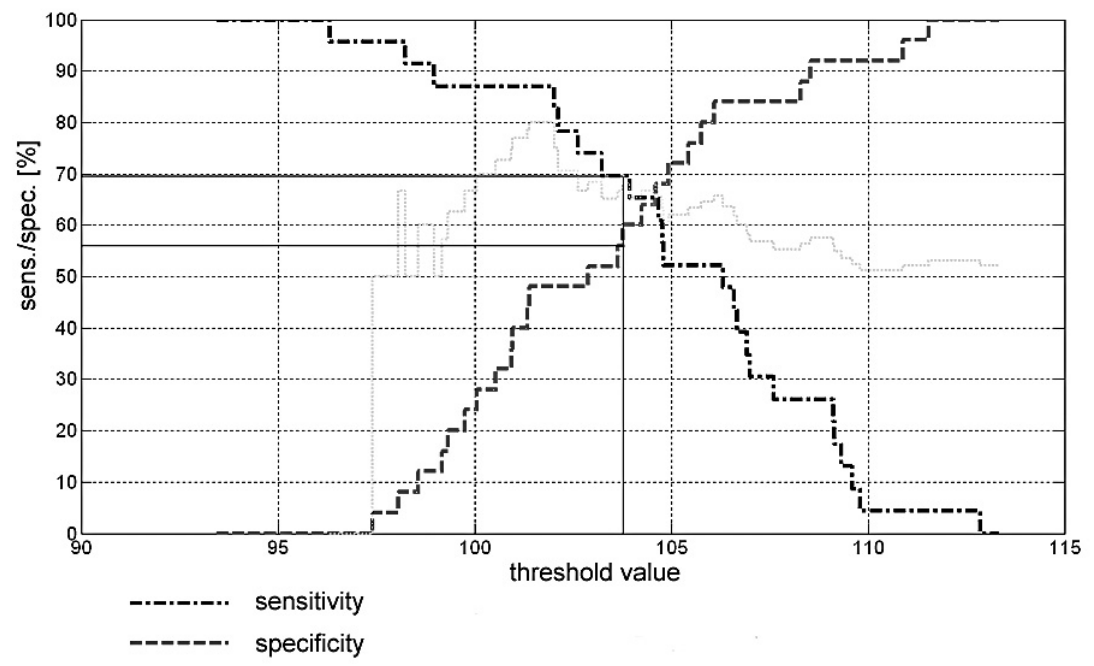

Fig. 2.Sensitivity and specificity in dependence on the threshold value

It is possible to use each of them individually, but the aim of classification is combine them to achieve better results. The features combination was performed by two methods based on average and weighted relative rank, and by so-called optimal weights and reduced optimal weights method inspired by neural networks. Combining the 12 good features we have obtained a set of new combined-features. 


\section{Results}

Mentioned methods of feature extraction and classification were tested on thermogram set, which consists of 140 thermograms. We have 30 thermograms of patients with confirmed breast cancer and 110 thermograms of healthy women in time of this paper submission. All methods were implemented in easy-to-use MATLAB environment.

The best feature found is homogeneity of cross co-occurrence matrix with intersection of specificity and sensitivity at $67 \%$. Combining features together further enhanced the results obtained by individual features - the best of them, optimal weights, reached intersection of specificity and sensitivity $79 \%$, and has $53 \%$ specificity for $90 \%$ sensitivity.

\section{Conclusions}

The thermogram classification results are comparable with commonly used diagnostic techniques (table1). X-Ray mammography, on which is based screening programme in Czech Republic, have a bit better results, but the results are strongly dependent on the radiologist and further, it employs dangerous radiation, which may caused new cancer genesis.

Tab. 1: Statistical parameters of commonly used diagnostic techniques

\begin{tabular}{|c|c|c|}
\hline Modality & Sensitivity & Specificity \\
\hline X-Ray Mammography & $80-90 \%$ & $65-85 \%$ \\
\hline Ultrasonography & $70-80 \%$ & $60 \%$ \\
\hline MRI systems & $80 \%$ & $70 \%$ \\
\hline DOBI (optical system) & $90 \%$ & $70 \%$ \\
\hline Thermography ) & $79 \%$ & $79 \%$ \\
\hline *the intersection of sensitivity and specificity of our best combined feature \\
\hline
\end{tabular}

Ultrasonography has low spatial resolution, which may be problem in early stage cancer detection. Examination on MRI system is very expansive. For thermography speaks, except the comparable results, its non-invasiveness, low expenses and applicability to young women where the mammography fails because of high density of breast tissue.

Our future research will be oriented deep analysis of correlation between the mammography, ultrasonography and thermography findings. The main task remains in development of new analysing and classification methods and algorithmization of commonly used classification systems [1]. We also need to extent our set of thermograms. We cooperate with mammography clinic, but we also plan to build interactive internet database of thermograms, which would be suitable for thermogram exchange. Eventually we are ready to join to some similar existing project.

\section{REFERENCES}

[1] AMALRICK, R., SPITALIER, J. M., GIRAUD, D., ALTSCHULER, C., Thermography in Diagnosis of Breast Diseases, , Thermography, Bibliotheca Radilogica, 1975, no. 6, p. 57 - 64, ISBN 3-8055-2134.

[2] DUB, P., Breast Cancer Detection Using Infrared Camera, PhD Thesis, Brno University of Technology, 2003

Acknowledgement: This project is supported by FRVS 2004/1599 